\title{
Cutoff points of T1 rho/T2 mapping relaxation times distinguishing early-stage and advanced osteoarthritis
}

Zhijian Yang ${ }^{1}$, Chao Xie'2, Songwen $\mathrm{Ou}^{2}$, Minning Zhao 3 , Zhaowei Lin²

\author{
'Department of Joint Surgery, First Affiliated Hospital of Sun Yat-sen University, \\ Guangzhou, Guangdong, China \\ 2Department of Orthopaedics, Zhujiang Hospital, Southern Medical University, \\ Guangzhou, Guangdong, China \\ 3Department of Radiology, Zhujiang Hospital, Southern Medical University, \\ Guangzhou, Guangdong, China
}

Submitted: 29 April 2021; Accepted: 1 August 2021

Online publication: 2 August 2021

Arch Med Sci 2022; 18 (4): 1004-1015

DOI: https://doi.org/10.5114/aoms/140714

Copyright @ 2021 Termedia \& Banach

\section{Abstract}

Introduction: The histopathology grading system is the gold standard post-operative method to evaluate cartilage degeneration in knee osteoarthritis (OA). Magnetic resonance imaging (MRI) T1 rho/T2 mapping imaging can be used for preoperative detection. An association between histopathology and T1 rho/T2 mapping relaxation times was suggested in previous research. However, the cutoff point was not determined among different histopathology grades. Our study aimed to determine the cutoff point of T1 rho/T2 mapping.

Material and methods: T1 rho/T2 mapping images were acquired from 80 samples before total knee replacements. Then the histopathology grading system was applied.

Results: The mean T1 rho/T2 mapping relaxation times of 80 samples were $39.17 \mathrm{~ms}$ and $37.98 \mathrm{~ms}$ respectively. Significant differences were found in T1 rho/T2 mapping values between early-stage and advanced OA ( $p<$ 0.001). The cutoff point for T1 rho was $33 \mathrm{~ms}$ with a sensitivity of 94.12 (95\% Cl: 80-99.3) and a specificity of 91.30 (95\% Cl: 79.2-97.6). The cutoff point for T2 mapping was suggested as $35.04 \mathrm{~ms}$ with a sensitivity of 88.24 (95\% Cl: $72.5-96.7)$ and specificity of 97.83 (95\% Cl: 88.5-99.9). After bootstrap simulation, the $95 \% \mathrm{Cl}$ of the $\mathrm{T} 1$ rho/T2 mapping cutoff point was estimated as 29.36 to $36.32 \mathrm{~ms}$ and 34.8 to $35.04 \mathrm{~ms}$ respectively. The area under the PR curve of T1 rho/T2 mapping was $0.972(95 \% \mathrm{Cl}: 0.925-0.992)$ and 0.949 (95\% Cl: 0.877-0.989) respectively.

Conclusions: The cutoff point of $\mathrm{T} 1$ rho relaxation times, which was suggested as $33 \mathrm{~ms}$, could be used to distinguish early-stage and advanced OA.

Key words: knee osteoarthritis, T1 rho, T2 mapping, histopathology.

\section{Introduction}

At present, histopathology is one of the most important methods to evaluate the degree of cartilage wear in knee osteoarthritis (OA). However, histopathology cannot be performed before total knee arthroplasty (TKA). Recently, relevant studies [1-8] indicated that magnetic resonance imaging (MRI) T1 rho/T2 mapping imaging could be used for preoperative detection. A positive association between the severity of cartilage degeneration and $\mathrm{T} 1$ rho/T2 mapping relaxation times was reported recently

\author{
Corresponding author: \\ Dr. Zhaowei Lin \\ Department \\ of Orthopaedics \\ Zhujiang Hospital \\ Southern Medical \\ University, Guangzhou \\ Guangdong, China \\ E-mail: linzhaowei0660@ \\ smu.edu.cn
}


[1-8]. Some studies [9-11] highlighted that T1 rho/ T2 mapping could be used to distinguish early OA and late OA. However, the cutoff point of the T1 rho/T2 mapping value has not been determined yet.

Therefore, in this study, 88 samples were obtained from 22 patients who underwent TKA. MRI T1 rho/T2 mapping sequences were performed preoperatively. The Osteoarthritis Research Society International (OARSI) grading system was employed post-operatively. After excluding OARSI grade 5 and grade 6 , eighty samples were collected for further evaluation. The OARSI grading system was applied to distinguish early-stage OA (OARSI grade 0-1) and advanced OA (OARSI grade 2-4). Eighty samples were divided into four femoral condyles: the medial femoral posterior condyle (MFPC), the lateral femoral posterior condyle (LFPC), the medial femoral distal condyle (MFDC), and the lateral femoral distal condyle (LFDC).

\section{Material and methods}

\section{Patients}

Twenty-two knee osteoarthritis (OA) patients were recruited before total knee arthroplasty. This research was approved and then supervised by the Ethical Committee of Zhujiang Hospital (trial register number: 2017-GJGBK-001).

\section{Magnetic resonance imaging (MRI)}

Before total knee arthroplasty, MRI was performed with a 3.0-T Achieva unit (Philips Healthcare, Eindhoven, The Netherlands). Fast spin-echo proton density-weighted fat suppression image (FSE PDFs) was performed based on the following parameters: repetition time $=1800 \mathrm{~ms}$, echo time $=35 \mathrm{~ms}$, layer thickness $=4 \mathrm{~mm}$, field of view $=180 \mathrm{~mm}$, matrix size $=320 \times 56$, excitation times $=2$, scanning layers $=20$, scanning time $=2 \mathrm{~min} 30 \mathrm{~s}$. The sagittal T1rho sequence was performed as follows: repetition time $=5.3 \mathrm{~ms}$, echo time $=0 \mathrm{~ms}$, optional locking time = $0 / 10 / 40 / 80 \mathrm{~ms}$, slice thickness $=4 \mathrm{~mm}$, slice thickness $=0.5 \mathrm{~mm}$, scanning layers $=18$, field of view $=160 \mathrm{~mm}$, matrix size $=256 \times 128$, excitation times $=1$, scanning time $=7 \mathrm{~min} 7 \mathrm{~s}$. The sagittal T2 mapping sequence was performed as follows: repetition time $=1000 \mathrm{~ms}$, echo time $=$ $0 \mathrm{~ms}$, optional locking time $=9.5 / 19.1 / 28.6 / 38.1 /$ $47.6 / 57.2 / 66.7 / 76.2 \mathrm{~ms}$, slice thickness $=4 \mathrm{~mm}$, slice thickness $=0.5 \mathrm{~mm}$, scanning layers $=20$ layers, field of view $=160 \mathrm{~mm}$, matrix size $=320$ $\times 256$, excitation times $=1$, scanning time $=8 \mathrm{~min}$ $46 \mathrm{~s}$. The following three-dimensional fat suppression (3D FSE cube) sequence was employed: $\mathrm{TR}$ time $=1500 \mathrm{~ms}$, repetition time $=25 \mathrm{~ms}$, slice thickness $=1.6 \mathrm{~mm}$, field of view $=160 \mathrm{~mm}$, matrix size $=256 \times 256$, excitation times $=1$, scanning time $=4 \mathrm{~min} 28 \mathrm{~s}$. T1 rho and T2 mapping relaxation times, whose units are millisecond (ms), were calculated while T2 mapping relaxation times were obtained.

\section{Total knee arthroplasty}

After 22 patients signed the consent form and completed the MRI scanning, total knee replacement was performed according to the general operating procedure. All condyles were resected into four groups: MFPC, LFPC, MFDC, and LFDC. All 88 samples were examined histologically.

\section{Histopathology}

Four kinds of femoral condyles (MFPC, LFPC, MFDC, and LFDC) were fixed in 4\% polymethyl alcohol, decalcified with Rapid Bone Decalcifier (American Master Tech Inc., Lodi, CA, USA) for 48 h, then embedded in paraffin. The femoral condyles were sagittally cut into slices with a thickness of $8 \mu \mathrm{m}$. All sections were stained with hematoxylin-eosin (HE) and safranin-fast green. Two pathologists (Songwen $\mathrm{Ou}$ and Chao $\mathrm{Xie}$ ) performed a routine histopathological evaluation of each section based on the OARSI osteoarthritis cartilage assessment system [12]. If different assessments were obtained, the results were discussed with a third pathologist (Zhaowei Lin) to reach a consensus and determine the final pathological grade.

\section{Image analysis}

All images were analyzed in a Philips DICOM Viewer R3.0-SP13 (Philips, Best, the Netherlands). The regions of interest (ROI) were drawn by a single radiologist (Minning Zhao) using each relaxation map. In sagittal images, each condyle was divided into four ROI parts: MFDC, LFDC, MFPC, and LFPC. The posterior edge of the anterior meniscal horn and the posterior edge of the posterior meniscal horn were used to segment the femoral distal condyle (FDC). The femoral posterior condyle was posterior to the posterior edge of the posterior meniscal horn (Figure 1). Among all sagittal images of the same ROI part from one sample, the image with the highest signal-to-noise ratio was selected for analysis [13]. T1 rho and T2 mapping relaxation time values are the average values of pixels (ms) in that selected ROI which reflected the relaxation times of T1 rho and T2 mapping. In horizontal images, the center reference line (c-line) was drawn through the deepest point of the groove and the femoral notch. The perpendicular distance ( $p$-distance) between selected images and the c-line was calculated by the number of slices in between (Figure 2). All MRI analyses were acquired before surgery. 

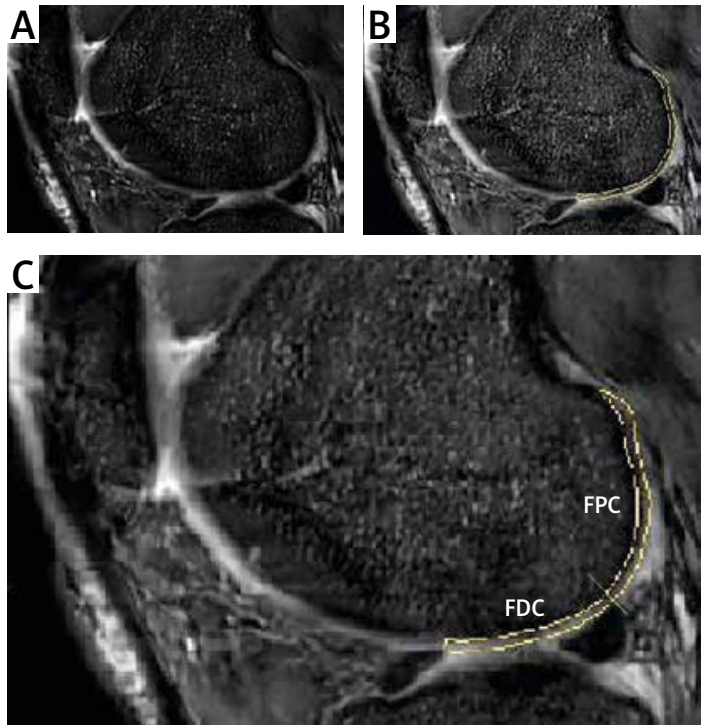
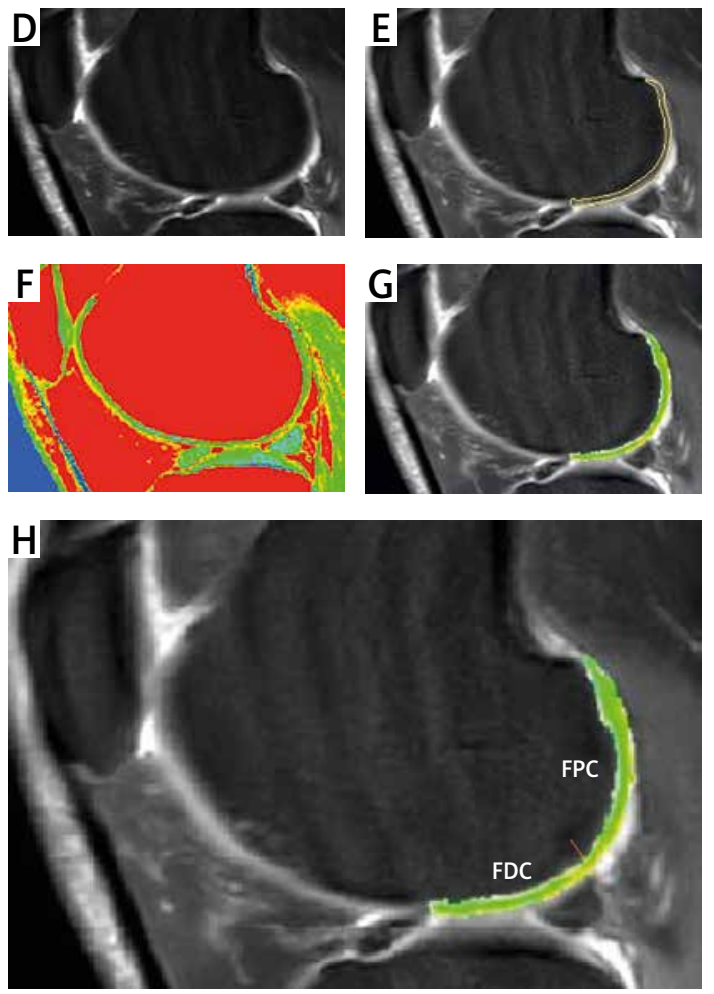

Figure 1. T1 rho/T2 mapping and ROls. A - Sagittal T1rho sequence was performed. B - The regions of interest (ROI) were drawn. The posterior edge of the anterior meniscal horn was used to separate the edge of ROI. C - ROI of condyles was divided into two parts: the femoral distal condyle and the femoral posterior condyle. The posterior edge of the posterior meniscal horn was used to segment the femoral distal condyle. D - T2 Fast spin-echo proton density-weighted fat suppression imaging (FSE PDFs) was performed. $\mathbf{E}-\mathrm{ROI}$ of T2 was drawn. F - A sagittal T2 mapping sequence was performed. G - T2 images were merged with the ROI of T2 mapping images. $\mathrm{H}-\mathrm{The}$ femoral distal condyle and the femoral posterior condyle were outlined

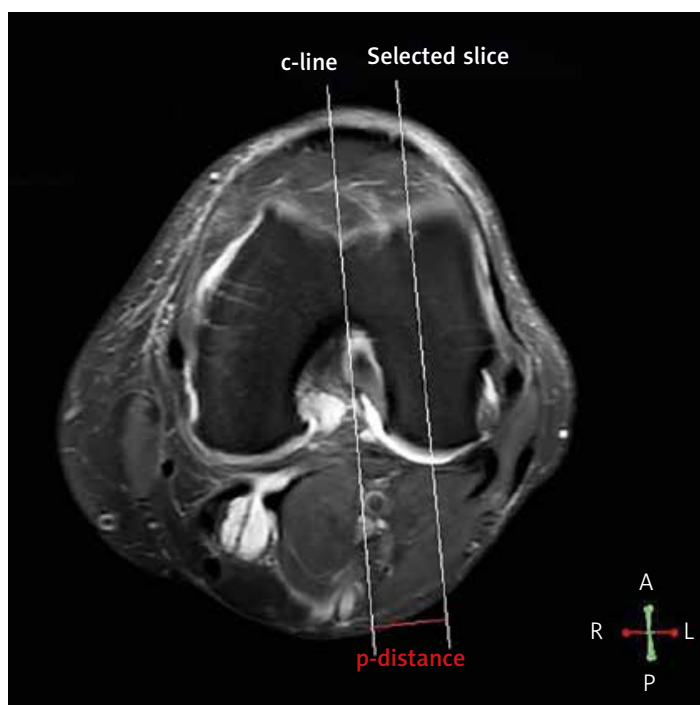

Figure 2. Reference lines were drawn in horizontal images. In horizontal MRI images, the center reference line (c-line) was drawn through the deepest point of the groove and the deepest point of the femoral notch. The reconstructed sagittal slices were parallel to the c-line. The perpendicular distance ( $p$-distance) between the selected slice (sagittal images) and c-line was calculated by multiplying the slice thickness by the number of slices in between

\section{ROIs of histopathology match ROIs of MRI}

The c-line of the condyle was drawn during surgery for surgery consideration. The ROI (R-line) of histopathology was drawn parallel to the c-line. P-distance was applied to determine the interval between the c-line and the R-line. The ROI was obtained and marked by the surgeon during surgery. Histopathological staining was employed post-operatively (Figure 3). Samples that were OARSI grade 5 or grade 6 were excluded since rare cartilage existed for MRI detection. Of 88 samples, 8 samples were OARSI grade 5 or grade 6 . Through the above method [14], ROls of histopathology were matched with ROIs of MRI theoretically (Figure 4). Eighty samples were obtained after excluding OARSI grade $5 / 6$.

\section{Statistical analysis}

All 80 samples were included for statistical analysis using MedCalc (MedCalc Software, Ostend, Belgium). Mean T1 rho and T2 mapping relaxation times (ms) with standard error (SE) were compared between early OA and advanced OA. The significance level was set as $p<0.05$. Receiver 
operating characteristic (ROC) analysis, area under the curve (AUC), potential cutoff points, sensitivity, specificity and their $95 \%$ confidence interval $(95 \% \mathrm{Cl})$, interactive dot diagrams, Youden index, misclassification-cost term (MCT) plot, and precision-recall (PR) curves were all described. In the ROC analysis, the area enclosed with the coordinate axes was 1.00. The area enclosed with the coordinate axis under the ROC curve was defined as AUC. The closer the AUC was to 1.00 , the higher the authenticity of the detection method. The certain accuracy level was set as AUC >0.9; the larger the AUC, the higher the diagnostic accuracy. The cutoff point of sensitivity-specificity curves provided a potential optimal diagnostic index for distinguishing between early-stage and advanced $\mathrm{OA}$. The sensitivity and specificity and their $95 \% \mathrm{Cl}$ were described as percentages. A potential cutoff point (ms) for distinguishing between early-stage OA and advanced OA was determined as the junction point of the sensitivity-specificity curve. The optimal criterion line was demonstrated in the interactive dot diagrams. The Youden index is the sum of sensitivity and specificity minus 1 (Youden index $=$ sensitivity + specificity -1 ). The highest point of the Youden index and the lowest point of the MCT curve were determined to further verify the reliability of the cutoff point (the junction point of the sensitivity-specificity curve). The bootstrap simulation was obtained using SPSS version 22 (IBM SPSS Software, Chicago, Illinois, USA). Based on the bootstrap simulation, the relaxation time data of T1 rho/T2 mapping were used for 10000 resampling simulations. After simulation, 95\% Cls of AUC, Youden index, and cutoff points were estimated. The PR curves of T1 rho and T2 mapping were analyzed together using the bootstrap simulation.

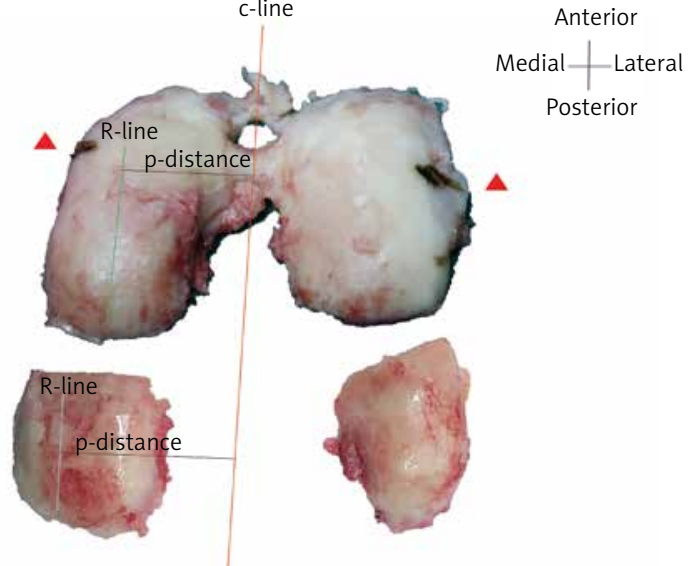

Figure 3. Reference lines were drawn in condyles. The center reference line (c-line) was drawn during surgery for surgery consideration. The ROI (R-line) of histopathology was drawn parallel to the c-line. P-distance was applied to determine the interval between the c-line and the R-line. The ROI was obtained and marked by the surgeon during surgery. Histopathological staining was employed in ROls

\section{Results}

\section{T1 rho and T2 mapping values of ROIs between early and advanced $O A$}

Of the 80 samples, 46 were early-stage OA, whereas the remainder had advanced OA. The sagittal T1 rho sequence was performed (Figure $1 \mathrm{~A}$ ) and the ROI were drawn (Figure $1 \mathrm{~B}$ ). The T2 image (FSE PDFs) and ROI of T2 were performed (Figures 1 D, E). The sagittal T2 mapping sequence was also performed (Figure $1 \mathrm{~F}$ ) and T2 images were merged with the ROI of T2 mapping images (Figure $1 \mathrm{G}$ ). The femoral distal condyle and the femoral posterior condyle were outlined separately in T1 rho images (Figure $1 \mathrm{C}$ ) and T2 mapping images (Figure $1 \mathrm{H}$ ). For the whole sample, the mean T1 rho relaxation time of 80 samples was $39.17 \mathrm{~ms}(95 \% \mathrm{Cl}$ : $33.72-44.62)$ while
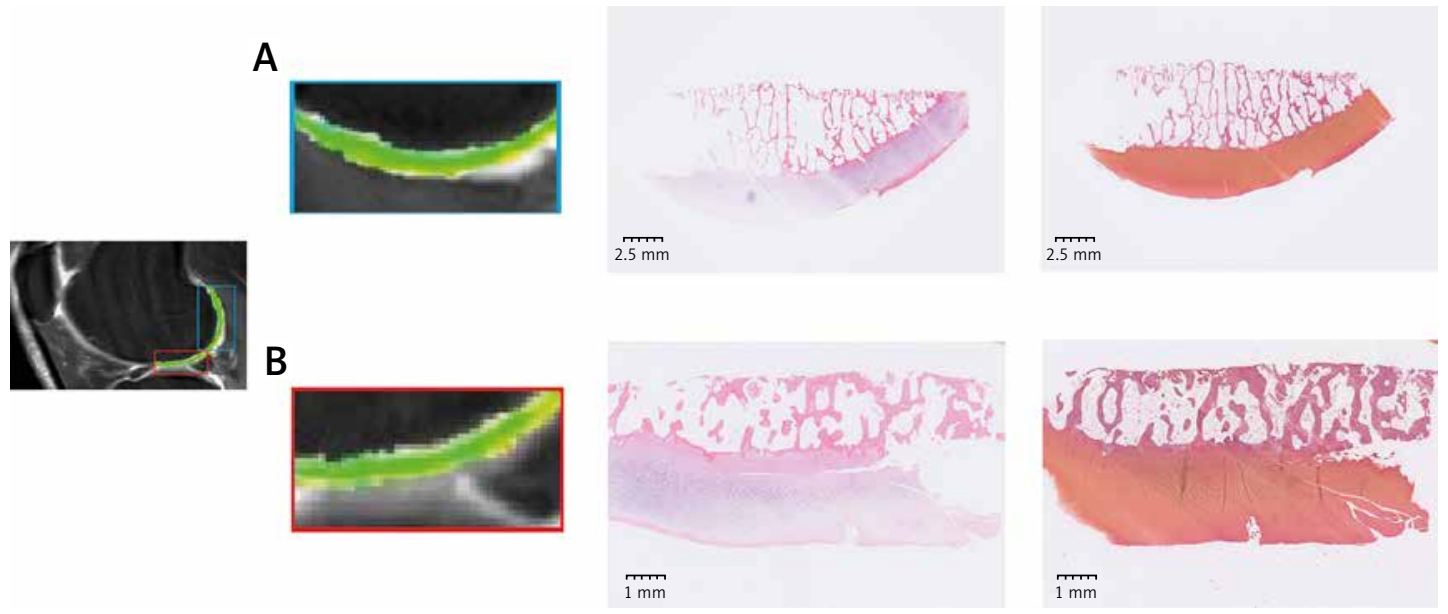

Figure 4. HE and safranin-fast green staining. A - The ROI showed the T2 mapping images of the femoral posterior condyle. HE staining and safranin-fast green staining were shown simultaneously. B - T2 mapping images, $\mathrm{HE}$ staining, and safranin-fast green staining of the femoral distal condyle were employed 
Table I. T1 rho and T2 mapping values of ROls between early and advanced OA

\begin{tabular}{|lcc|}
\hline Variable & $\begin{array}{c}\text { T1 rho relaxation } \\
\text { times [ms] }\end{array}$ & $\begin{array}{c}\text { T2 mapping } \\
\text { relaxation times } \\
\text { [ms] }\end{array}$ \\
\hline 80 samples & $39.17 \pm 2.74$ & $37.98 \pm 2.00$ \\
\hline Early-stage OA & $25.20 \pm 0.87$ & $29.81 \pm 0.52$ \\
\hline Advanced OA & $58.08 \pm 4.69$ & $49.03 \pm 3.96$ \\
\hline
\end{tabular}

the mean $\mathrm{T} 2$ mapping relaxation time of all samples was $37.98 \mathrm{~ms}$ (95\% Cl: 33.99-41.97). A significant difference was found in T1 rho/T2 mapping values between early-stage and advanced OA $(p<0.001)$ (Table I, Figure 5). Histograms of T1 rho/T2 mapping values classified as OARSI grades (grade 1-4) or early/advanced OA are shown in Figure 6.

\section{ROC curves and cutoff points}

Figure $7 \mathrm{~A}$ and $8 \mathrm{~A}$ display the ROC curves which were used to distinguish between early $\mathrm{OA}$ and advanced OA. In Figure $7 \mathrm{~A}$ or $8 \mathrm{~A}$, the area below the ROC curve (the blue curve) was defined as AUC. AUCs of these two ROC curves were both $>0.9$ (Tables II and III). Figures $7 \mathrm{~B}$ and $8 \mathrm{~B}$ show the overlapping sensitivity-specificity curves. The point of intersection was suggested as the optimal criterion for differentiating early-stage and advanced OA. Interactive dot diagrams of early-stage OA and advanced $\mathrm{OA}$ are shown with each optimal criterion line (Figures $7 \mathrm{C}, 8 \mathrm{C}$ ). Additionally, the Youden index was estimated (Figures 7 D, 8 D). The non-parametric method suggested a cutoff point at $33 \mathrm{~ms}$ for T1 rho with the sensitivity of 94.12 (95\% Cl: 80.3-99.3) and specificity of 91.30 (95\% Cl: 79.2-97.6) (Table II). The cutoff point for T2 mapping was 35.04 ms with a sensitivity of $88.24(95 \% \mathrm{Cl}: 72.5-96.7)$ and specificity of $97.83(95 \% \mathrm{Cl}: 88.5-99.9)$ (Table III). Precision recall (PR) curves of $\mathrm{T} 1$ rho and $\mathrm{T} 2$ mapping are also shown in Figures $7 \mathrm{E}$ and $8 \mathrm{E}$. Figures $7 \mathrm{~F}$ and $8 \mathrm{~F}$ show the MCT plot with the lowest point on this curve corresponding to the optimal criterion. The highest point of the Youden index (Figures 7 D, 8 D) was at the same optimal criterion.

\section{Bootstrap simulation}

The bootstrap method was used to simulate ROC analysis which would determine the variable estimation of the ROC cutoff point. Ten thousand resamples, which were all different random number seeds, were simulated from original data of T1 rho and T2 mapping. After simulation, the $95 \% \mathrm{Cl}$ of T1 rho/T2 mapping AUC was suggested in Tables IV and $\mathrm{V}$. The $95 \% \mathrm{Cl}$ of $\mathrm{T} 1$ rho/T2 mapping Youden index was also analyzed (Tables IV and V). After bootstrap simulation, the $95 \% \mathrm{Cl}$ of the $\mathrm{T} 1$ rho cutoff point was estimated as 29.36 to 36.32 $\mathrm{ms}$ and the $95 \% \mathrm{Cl}$ of the $\mathrm{T} 2$ mapping cutoff point was estimated as 34.8 to $35.04 \mathrm{~ms}$.

Precision-recall curves of T1 rho and T2 mapping were analyzed after bootstrap simulation (Figure 9). The area under the PR curve of T1 rho was significantly greater than that of $\mathrm{T} 2$ mapping (Table VI). The difference between these two areas was estimated at 0.02314 .

\section{Discussion}

The histopathology assessment system of cartilage is the gold standard to evaluate cartilage damage $[12,15]$. The Histologic/Histochemical Grading System (HHGS) was established to evaluate the quality of cartilage. However, this grad-
A

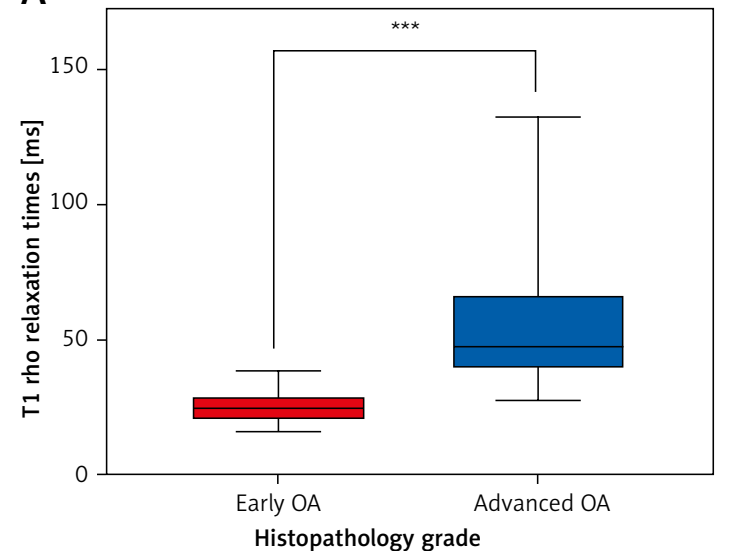

B

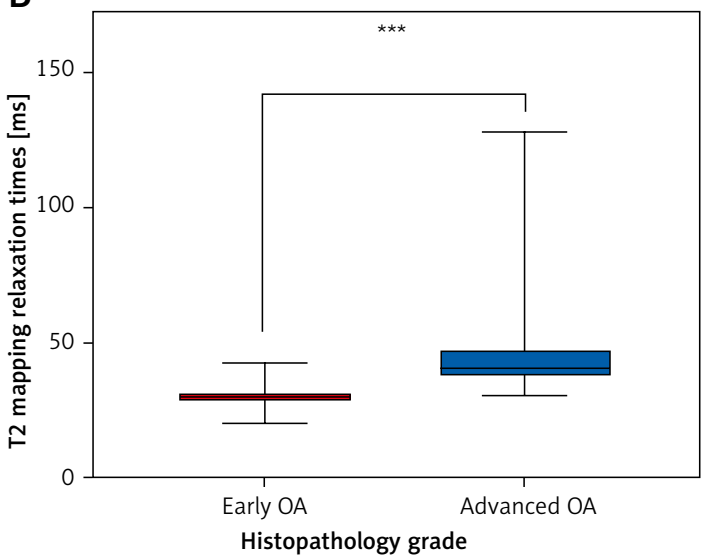

Figure 5. Mean relaxation times between early OA and advanced OA. A - T1 rho relaxation time values (ms) of 80 samples were divided into early OA and advanced OA. A significant difference was found between early-stage and advanced OA $(p<0.001)$. B - T2 mapping relaxation time values (ms) of 80 samples were divided into early OA and advanced OA. A significant difference was found between early and advanced OA $(p<0.001)$ 
A

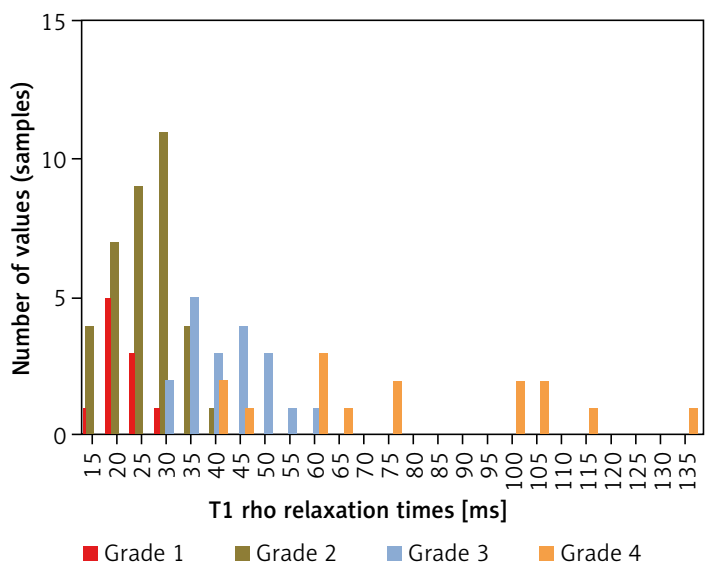

C

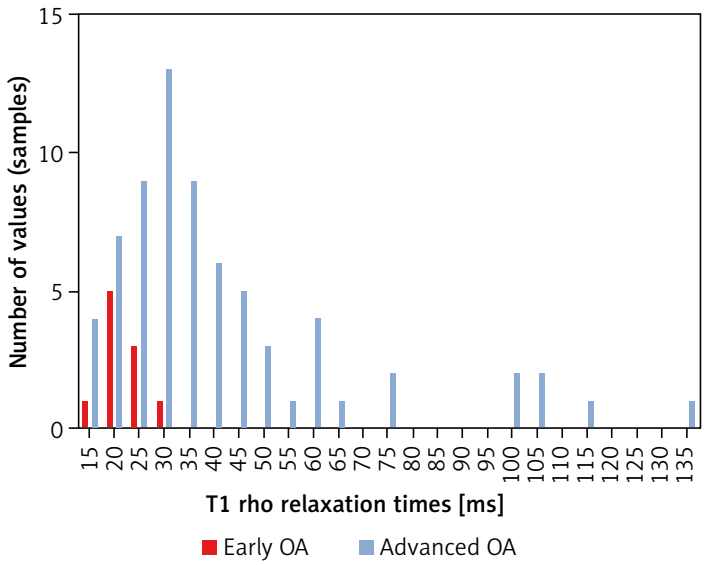

B
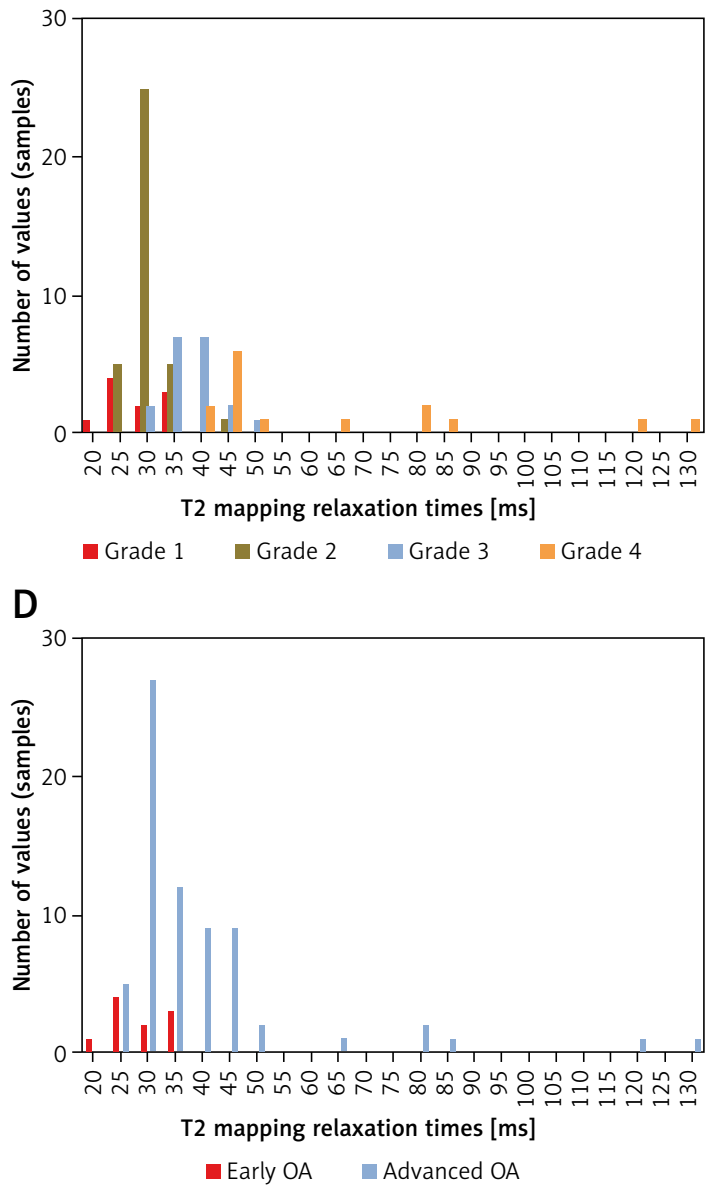

Figure 6. Histograms of relaxation times of different histopathology. A - Histograms of T1 rho relaxation times values (ms) were classified based on OARSI grades. B - Histograms of T2 mapping relaxation times values (ms) were classified based on OARSI grades. $\mathbf{C}$ - Histograms of $\mathrm{T} 1$ rho relaxation times values (ms) were divided into early $\mathrm{OA}$ and advanced OA. D - Histograms of T2 mapping relaxation time values (ms) were divided into early $\mathrm{OA}$ and advanced OA

ing system was established from well-advanced OA cartilage samples. Early-stage OA could not be evaluated properly by HHGS [16]. Thus, the Osteoarthritis Research Society International (OARSI) Cartilage Histopathology Assessment System (OOCHAS) system is recommended for studies investigating the association between histopathology and other parameters $[16,17]$. The OARSI system (OOCHAS system) included a grade methodology, stage assessment and scoring method. The OARSI grade methodology illustrated depth-damaging progression into cartilage, including grades $0-6$. The OARSI stage assessment illustrated the extent of joint involvement and scoring of points was the result of the grade times stage. A previous study [17] assumed that OA involving deeper cartilage degeneration was much more advanced and a good indicator of progressive disease. OARSI stage assessment was based on the horizontal extent of the injured cartilage surface and was categorized by percentage. However, the image analysis method [2] or post-operative guide- line of dividing condyles into different sections could not ensure that each sample had an equal horizontal extent, which might affect the denominator of the percentage. As a result, we considered that the OARSI stage was a bias in this case. Therefore, we chose the OARSI grade methodology as the post-operative gold standard of cartilage damage. Considering that there is sclerotic bone or reparative tissue but no cartilage involvement in OARSI grade 5 and grade 6 , it was presumed to be incapable of measuring T1rho/T2 mapping in MRI.

While cartilage edema and irregularity occurred in the superficial layer of cartilage in OARSI grade 1 , and fibrillation occurred in OARSI grade 2 , the microstructure and mechanical properties of the perichondral matrix changed. A previous study [12] showed significant differences in biomechanical properties between OARSI grade 1 and grade 2 , while no significant differences were shown in aggregate modulus from OARSI grade 2 to grade 5. No visible superficial degeneration existed in OARSI grade 1 cartilage; however, superficial fibril- 
A

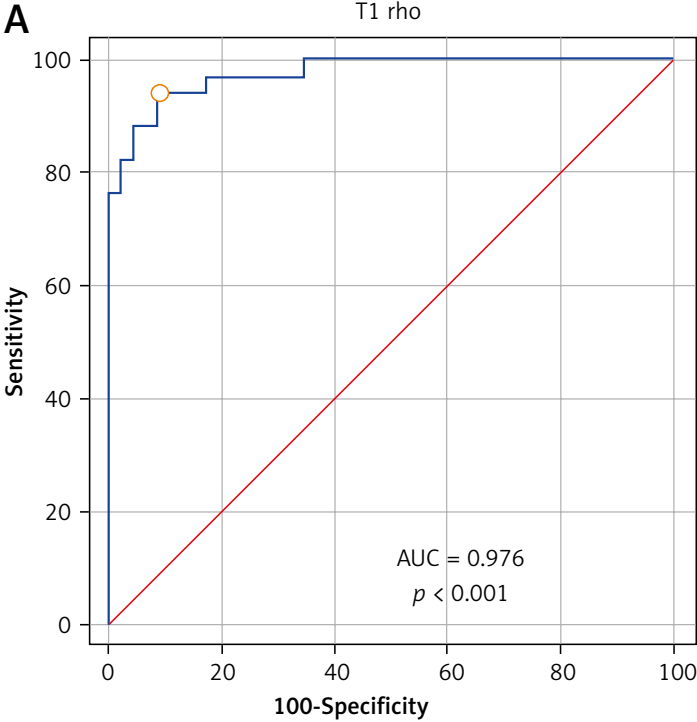

C

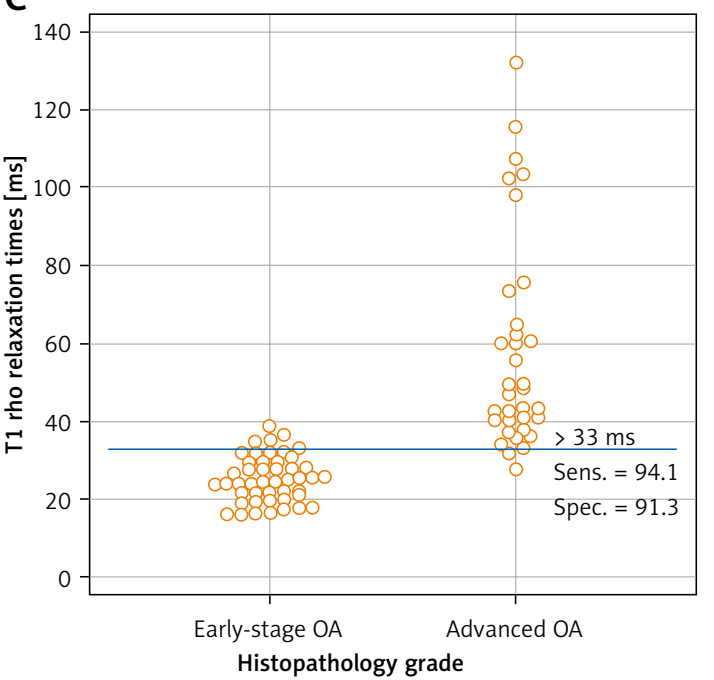

$\mathrm{E}$

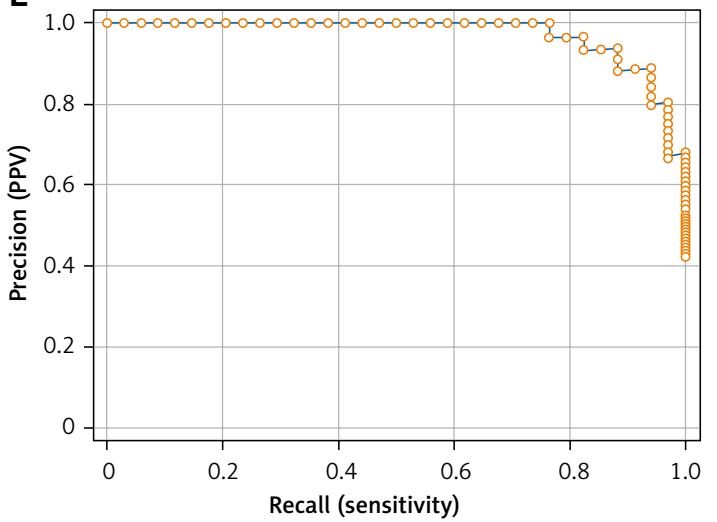

B

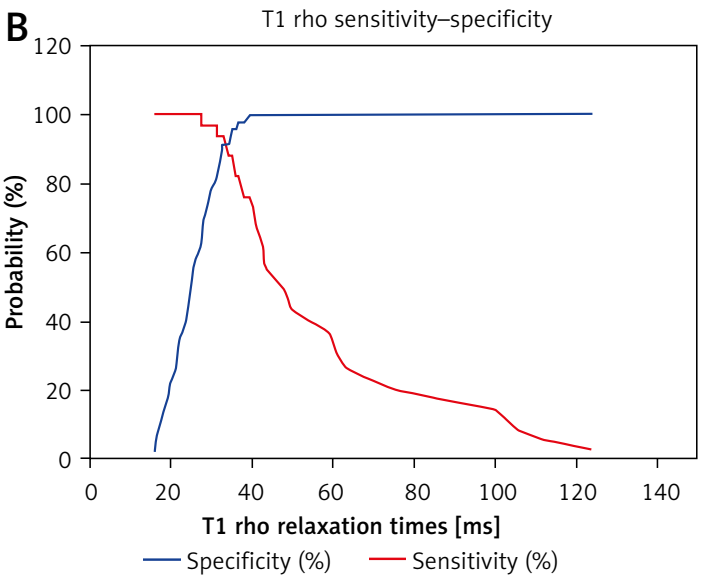

D

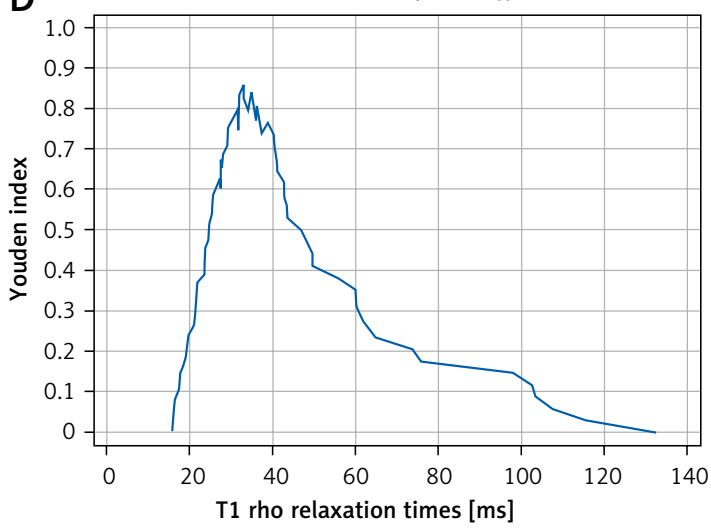

$\mathrm{F}$

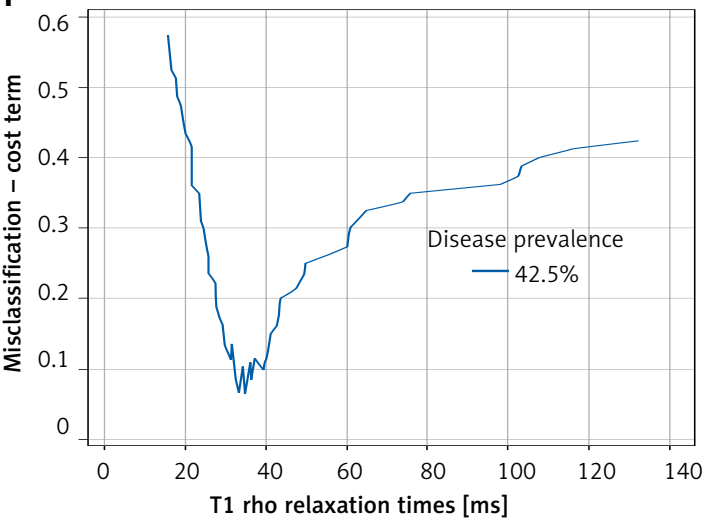

Figure 7. Receiver operating characteristic (ROC) curve and analysis of T1 rho. A - ROC curve of T1 rho relaxation times was established to find out the optimal criterion to distinguish mild OA and severe OA. Area under curve (AUC) was 0.976 (95\% Cl: 0.914-0.997, SE = 0.0139, $p<0.001)$. B - Sensitivity curve and specificity curve were intersected. The point of intersection was suggested as the optimal criterion to distinguish early OA and advanced OA. C - Interactive dot diagrams of early-stage OA and advanced OA were displayed with optimal criterion lines. D - Youden index was estimated. The highest point of the Youden index was at the same optimal criterion as the intersection point of the sensitivity-specificity curve. E - Precision recall (PR) curves of T1 rho relaxation times (ms) of 80 samples were also shown. $\mathbf{F}$ - The misclassification-cost term (MCT) plot showed that the lowest point corresponded to the optimal criterion 
A

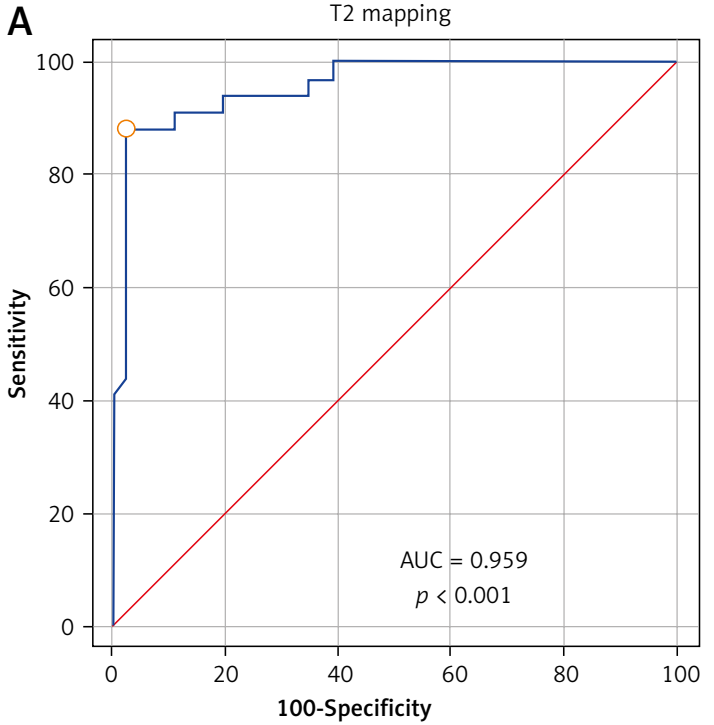

C

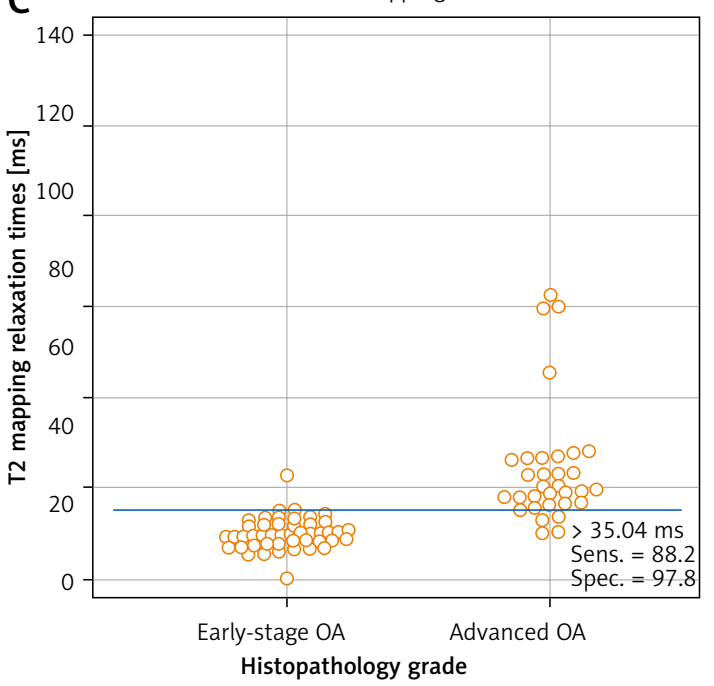

E

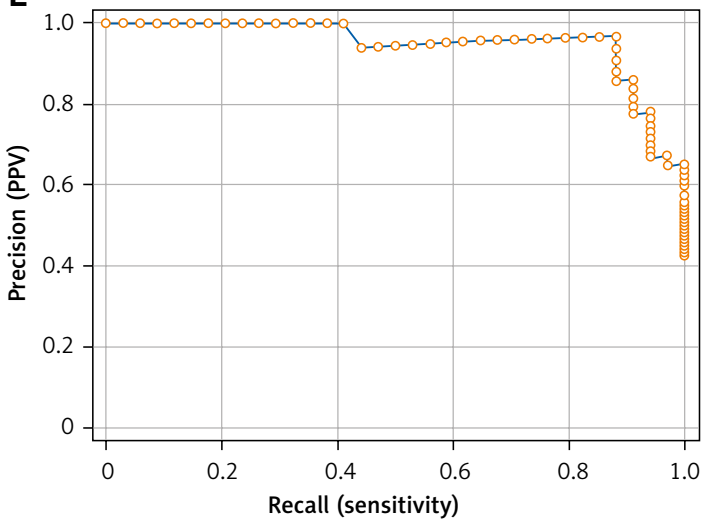

$\mathrm{B}_{120}$

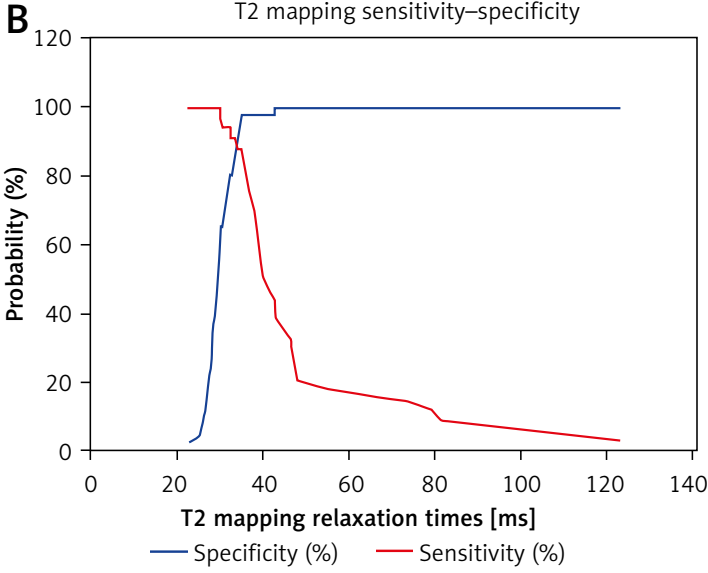

D

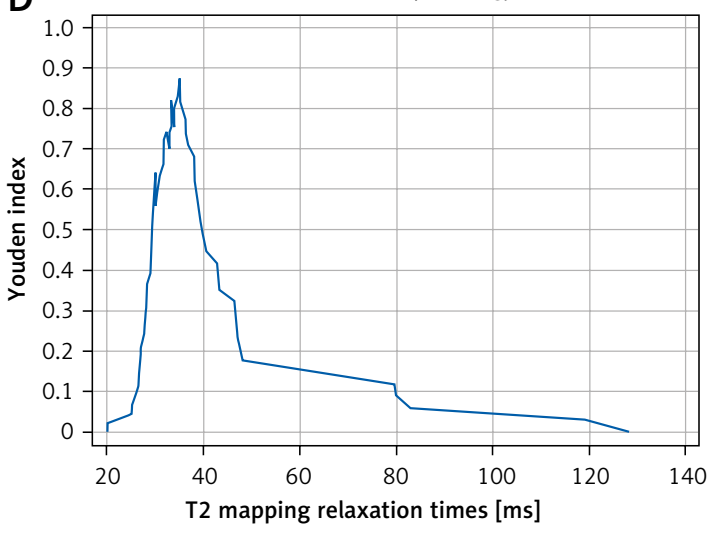

F Classification: histopathology

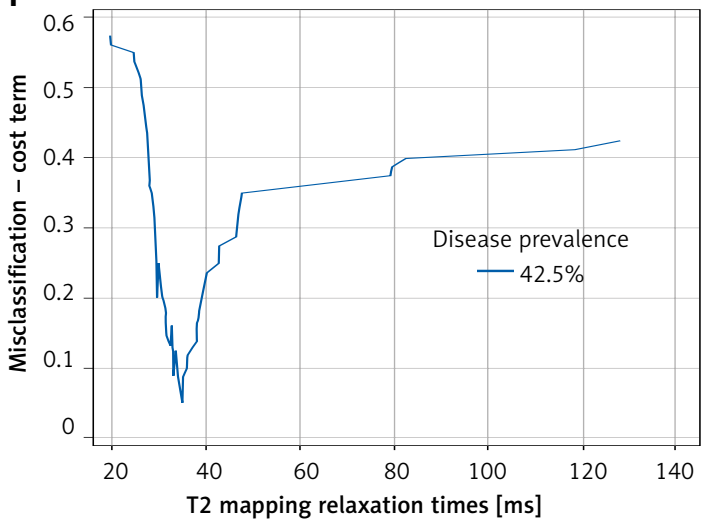

Figure 8. Receiver operating characteristic (ROC) curve and analysis of T2 mapping. A - ROC curve of T2 mapping was used to determine the distinguishing criterion between early-stage OA and advanced OA. AUC of ROC curve was 0.959 (95\% Cl: $0.890-0.991, \mathrm{SE}=0.0206, p<0.001)$. B - The sensitivity curve and specificity curves are displayed for estimation of the optimal criterion of the ROC curve which was the intersection point of the sensitivity-specificity curve. C - Interactive dot diagrams of early OA and advanced OA are displayed with optimal criterion lines. $\mathbf{D}$ - The highest point of the Youden index was at the same optimal criterion. $\mathbf{E}$ - Precision recall (PR) curves of T2 mapping are also shown. F - The lowest point of the misclassification-cost term (MCT) plot was estimated at the same point as the optimal criterion 
Table II. Area under the ROC curve (AUC) and Youden index of $\mathrm{T} 1$ rho

\begin{tabular}{|lc|}
\hline Parameter & Value \\
\hline Area under the ROC curve (AUC) & 0.976 \\
\hline Standard error $^{\mathrm{a}}$ & 0.0139 \\
\hline 95\% Confidence interval ${ }^{\mathrm{b}}$ & $0.914-0.997$ \\
\hline z statistic & 34.296 \\
\hline Significance level P (area $=0.5)$ & $<0.0001$ \\
\hline Youden index J & 0.8542 \\
\hline Associated criterion [ms] & $>33$ \\
\hline Sensitivity & 94.12 \\
\hline Specificity & 91.30 \\
\hline
\end{tabular}

aHanley \& McNeil, 1982, ${ }^{b}$ Binomial exact.

Table IV. Area under the ROC curve (AUC), Youden index and optimal criterion of T1 rho

\begin{tabular}{|c|c|}
\hline Parameter & Value \\
\hline Area under the ROC curve (AUC) & 0.976 \\
\hline Standard error ${ }^{a}$ & 0.0137 \\
\hline $95 \%$ Confidence interval $^{\mathrm{b}}$ & $0.914-0.997$ \\
\hline $95 \%$ Bootstrap $\mathrm{Cl}^{\mathrm{c}}$ & 0.928 to 0.993 \\
\hline z statistic & 34.818 \\
\hline Significance level P (area $=0.5)$ & $<0.0001$ \\
\hline Youden index J & 0.8542 \\
\hline $95 \%$ Confidence interval ${ }^{a}$ & $0.6995-0.9271$ \\
\hline Associated criterion [ms] & $>33$ \\
\hline $95 \%$ Confidence interval ${ }^{a}$ & $\begin{array}{c}>28.78178319 \text { to } \\
>36.32\end{array}$ \\
\hline Sensitivity & 94.12 \\
\hline Specificity & 91.30 \\
\hline Optimal criteriond $[\mathrm{ms}]$ & $>33$ \\
\hline $95 \%$ Confidence interval ${ }^{\mathrm{b}}[\mathrm{ms}]$ & $\begin{array}{l}>29.36 \text { to } \\
>36.32\end{array}$ \\
\hline Sensitivity & 94.12 \\
\hline Specificity & 91.30 \\
\hline
\end{tabular}

${ }^{a}$ Delong et al., $1988,{ }^{b}$ Binomial exact, ${ }^{c} B C_{a}$ bootstrap confidence interval (10 000 iterations; random number seed: 10 000), ${ }^{d}$ Taking into account disease prevalence (42.5\%) and estimated costs: cost False Positive: 1; cost False Negative: 1, cost True Positive: 0; cost True Negative: 0 .

lation, cell death, and mechanical changes existed simultaneously. Preoperative detection of these changes might provide an appropriate opportunity to take precautions against the pathological progress of osteoarthritis. More notably, the composition of the extracellular matrix of OARSI grade 2 cartilage had changed [18, 19], especially the collagen fiber arrangement. Many in vitro studies [20-22] regard the treatment before degeneration of extracellular matrix and superficial fissure as the key for early treatment of knee osteoarthritis. The lack of a preoperative imaging cutoff point
Table III. Area under the ROC curve (AUC) and Youden index of T2 mapping

\begin{tabular}{|lc|}
\hline Parameter & Value \\
\hline Area under the ROC curve (AUC) & 0.959 \\
\hline Standard error $^{\mathrm{a}}$ & 0.0206 \\
\hline 95\% Confidence interval $^{\mathrm{b}}$ & 0.890 to 0.991 \\
\hline z statistic & 22.267 \\
\hline Significance level P (area $=0.5)$ & $<0.0001$ \\
\hline Youden index J & 0.8606 \\
\hline Associated criterion [ms] & $>35.04$ \\
\hline Sensitivity & 88.24 \\
\hline Specificity & 97.83 \\
\hline
\end{tabular}

${ }^{a}$ Hanley \& McNeil, 1982, ${ }^{b}$ Binomial exact.

Table V. Area under the ROC curve (AUC), Youden index and optimal criterion of T2 mapping

\begin{tabular}{|c|c|}
\hline Parameter & Value \\
\hline Area under the ROC curve (AUC) & 0.959 \\
\hline Standard error ${ }^{a}$ & 0.0205 \\
\hline $95 \%$ Confidence interval ${ }^{b}$ & $0.890-0.991$ \\
\hline $95 \%$ Bootstrap $\mathrm{Cl}^{\mathrm{c}}$ & 0.898 to 0.988 \\
\hline z statistic & 22.363 \\
\hline Significance level $\mathrm{P}($ area $=0.5)$ & $<0.0001$ \\
\hline Youden index J & 0.8606 \\
\hline $95 \%$ Confidence interval ${ }^{a}$ & $0.7136-0.9488$ \\
\hline Associated criterion [ms] & $>35.04$ \\
\hline 95\% Confidence interval ${ }^{a}$ & $>34.8$ to $>35.04$ \\
\hline Sensitivity & 88.24 \\
\hline Specificity & 97.83 \\
\hline Optimal criterion ${ }^{\mathrm{d}}[\mathrm{ms}]$ & $>35.04$ \\
\hline $95 \%$ Confidence interval $^{\mathrm{b}}[\mathrm{ms}]$ & $>34.8$ to $>35.04$ \\
\hline Sensitivity & 88.24 \\
\hline Specificity & 97.83 \\
\hline
\end{tabular}

${ }^{a}$ DeLong et al., $1988,{ }^{b}$ Binomial exact, ${ }^{c} B C_{a}$ bootstrap confidence interval (10 000 iterations; random number seed: 10 000), ${ }^{d}$ Taking into account disease prevalence (42.5\%) and estimated costs: cost False Positive: 1; cost False Negative: 1, cost True Positive: 0; cost True Negative: 0 .

was the key issue hindering the application of this research in patients. Hence, the earlier OARSI grade $1 / 2$ is identified, the more opportune it is to prevent pathological progression.

However, in general, the criteria of early-stage OA included cartilage degeneration, which reflected OARSI grade $3-4[10,11,23]$. To identify an earlier stage of $\mathrm{OA}$, which could demonstrate the changes of microstructure and mechanical properties, OARSI grade 0/1 was defined as early-stage OA while OARSI grade 2-4 was considered as advanced $\mathrm{OA}$, which all could be measured by $\mathrm{T} 1$ 


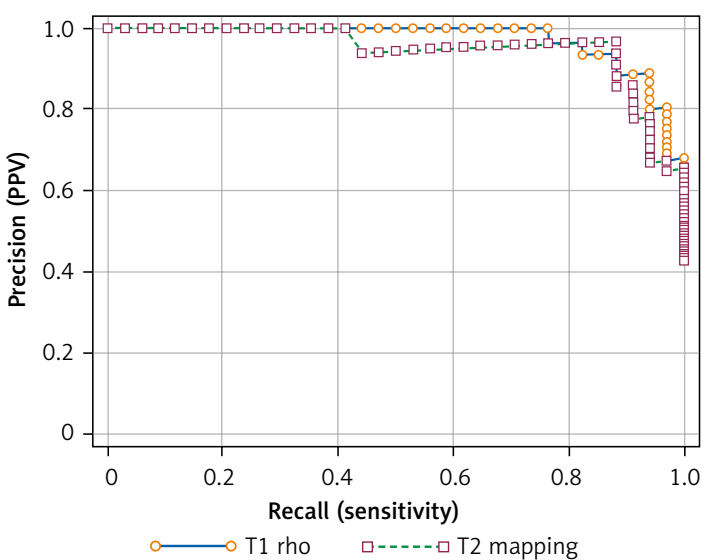

Figure 9. Precision-recall curve of $\mathrm{T} 1$ rho and $\mathrm{T} 2$ mapping. A bootstrap simulation was applied to assess the reliability of optimal criteria between $\mathrm{T} 1$ rho and T2 mapping. The area under the PR curve of $\mathrm{T} 1$ rho was significantly greater than that of $\mathrm{T} 2$ mapping, which suggested that the optimal criterion of T1 rho might be more reliable

rho/T2 mapping. This kind of classification was essential to be applied to explore the possibility of T1 rho/T2 mapping in detecting different changes of cartilage that could not be detected by morphological MRI, but whose mechanical structure had changed significantly.

Palmer's study [10] demonstrated that morphological MRI could identify OARSI grade 3; however, these routine knee MRI sequences were not sufficient for the assessment of earlier-stage OA. Physiologic imaging techniques such as T1 rho/ T2 mapping were needed for the evaluation of early-stage cartilage abnormalities (OARSI grade 0/1). T1 rho/T2 mapping could be used to reflect the proteoglycan or collagen fiber arrangement and water content in the early stage of cartilage. T1rho could describe the spin-lattice relaxation, thus reflecting the interaction effect between proteoglycan content in the cartilage extracellular matrix and the motion-restricted water molecules [1]. Previous studies [24, 25] suggested that the relaxation time value of $\mathrm{T} 1$ rho was related to the arrangement of collagen fibers in the extracellular matrix. Nishioka [26] found that T2 mapping was mainly affected by the changes in collagen fiber and water arrangement. Relevant literature [9, 27-29] also confirmed that T2 mapping and T1 rho measurements could be used to determine whether articular cartilage was damaged. Current T1 rho/T2 mapping studies have focused on comparing $\mathrm{T} 2$ and $\mathrm{T} 1$ rho relaxation times between different OA clinical grades. However, few studies have focused on discussing T1 rho/T2 mapping relaxation time cutoff points of different OARSI grades of OA. A key cutoff point that could discern the mechanical changes and microstructure changes with no visible superficial degeneration
Table VI. Comparison of precision-recall curves

\begin{tabular}{|lc|}
\hline Parameter & Value \\
\hline Variable & T1rho \\
\hline Area under curve & 0.972 \\
\hline $95 \%$ Bootstrap Cla & 0.925 to 0.992 \\
\hline F1 ${ }_{\text {max }}$ & 0.914 \\
\hline Associated criterion [ms] & $>33$ \\
\hline Variable & T2mapping \\
\hline Area under curve & 0.949 \\
\hline $95 \%$ Bootstrap Cl & 0.877 to 0.989 \\
\hline F1 ${ }_{\text {max }}$ & 0.923 \\
\hline Associated criterion [ms] & $>35.04$ \\
\hline Difference between areas & 0.02314 \\
\hline $95 \%$ Bootstrap Cla & $4.8298 E-16$ to 0.07229 \\
\hline
\end{tabular}

${ }^{a} B C_{a}$ bootstrap confidence interval (10 000 iterations; random number seed: 10 000).

was important for in vitro experiments and clinical treatment. Although some mechanical tools could be used to detect microstructural changes, it was impossible to apply them preoperatively. Based on this, our study further analyzed the specific cutoff point of T1 rho/T2 mapping which could be used to judge the severity of cartilage damage before surgery, especially to distinguish edema cartilage and fibrillation cartilage with biomechanical changes.

To identify the $95 \%$ confidence intervals the resample method should be used given that $\mathrm{T} 1 \mathrm{rho} /$ T2 mapping could not be applied in such a large number of patients due to its cost. Through bootstrap (Monte Carlo) simulation, multiple independent random samples could be obtained from the original data, and the $95 \%$ confidence interval of the cutoff point could be estimated. Given this large number of replicates (10 000 random bootstrap simulation resamples in our study), 95\% confidence intervals could be measured. We also compared the reliability of the cutoff point of $\mathrm{T} 1$ rho and T2 mapping. We further found that the measurement of T1 rho rather than T2 mapping could reveal the subtle changes of cartilage histopathologically.

These findings could play an important role in the earlier recognition and treatment of osteoarthritis. The cutoff point of T1 rho might be used as the key implication for the mechanical and microstructure changes of OA cartilage. At the same time, for physicians, early recognition of OA cartilage before superficial degeneration could be beneficial to patients. Furthermore, the T1 rho cutoff point could be key to determine which patients may benefit from preservation strategies and which are better candidates for joint surgery 
(since arthroscopy or joint replacement is applied for cartilage morphological degeneration). In addition, this diagnostic method may be used to develop personalized treatment for patients. For example, it might decide what kind of exercise or rehabilitation strategy should be adopted in the early stage to delay the progress of osteoarthritis and preserve the integrity of cartilage structure.

This study is not without limitations. The cutoff point of $\mathrm{T} 1 \mathrm{rho} / \mathrm{T} 2$ mapping could only distinguish the difference between OARSI grade 1 and OARSI grade 2 . To identify the occurrence of OA earlier, more updated non-invasive tools are needed in the future. In general, changes of OARSI grade 1 cartilage were more in the microstructure and chondrocytes. At the same time, the onset of knee osteoarthritis was not determined. The debate about knee OA originating from the cartilage or the subchondral bone is ongoing. More updated preoperative techniques are required to identify these onset changes microstructurally. Since the detection of $\mathrm{T} 1$ rho/T2 mapping itself could be performed in the existing MRI, it was of great significance for its promotion among hospitals. However, this technology itself was not prevalent, which may be a limitation. Only 80 samples undergoing TKA were employed in this study. Although the Monte Carlo method was used for bootstrap simulation, a larger sample size might be needed to consolidate our conclusions. Further long-term follow-up prospective clinical research is needed.

In conclusion, the cutoff point of the mean T1 rho relaxation time value (>33 ms) might be used as the measurement for identifying advanced OA. T1 rho relaxation times might be a better measurement than $\mathrm{T} 2$.

\section{Acknowledgments}

Zhijian Yang and Chao Xie contributed equally to this study.

\section{Conflict of interest}

The authors declare no conflict of interest.

\section{References}

1. Li X, Benjamin Ma C, Link TM, et al. In vivo T(1rho) and $T(2)$ mapping of articular cartilage in osteoarthritis of the knee using 3 T MRI. Osteoarthritis Cartilage 2007; 15: 789-97.

2. Kester BS, Carpenter PM, Yu HJ, et al. T1 $\mathrm{\rho} / \mathrm{T} 2$ mapping and histopathology of degenerative cartilage in advanced knee osteoarthritis. World J Orthop 2017; 8: 350-6.

3. Monu UD, Jordan CD, Samuelson BL, Hargreaves BA, Gold GE, McWalter EJ. Cluster analysis of quantitative MRI T2 and T1 $\rho$ relaxation times of cartilage identifies differences between healthy and ACL-injured individuals at 3T. Osteoarthritis Cartilage 2017; 25: 513-20.
4. Mahar R, Batool S, Badar F, Xia Y. Quantitative measurement of T2, T1 $\rho$ and $T 1$ relaxation times in articular cartilage and cartilage-bone interface by SE and UTE imaging at microscopic resolution. J Magn Reson 2018; 297: 76-85.

5. Urish KL, Keffalas MG, Durkin JR, Miller DJ, Chu CR, Mosher TJ. T2 texture index of cartilage can predict early symptomatic OA progression: data from the osteoarthritis initiative. Osteoarthritis Cartilage 2013; 21: 1550-7.

6. Williams A, Winalski CS, Chu CR. Early articular cartilage MRI T2 changes after anterior cruciate ligament reconstruction correlate with later changes in T2 and cartilage thickness. J Orthop Res 2017; 35: 699-706.

7. Wu Y, Yang R, Jia S, Li Z, Zhou Z, Lou T. Computer-aided diagnosis of early knee osteoarthritis based on MRI T2 mapping. Biomed Mater Eng 2014; 24: 3379-88.

8. Zhong H, Miller DJ, Urish KL. T2 map signal variation predicts symptomatic osteoarthritis progression: data from the Osteoarthritis Initiative. Skeletal Radiol 2016; 45: 909-13.

9. Carballido-Gamio J, Stahl R, Blumenkrantz G, Romero A, Majumdar S, Link TM. Spatial analysis of magnetic resonance T1rho and T2 relaxation times improves classification between subjects with and without osteoarthritis. Med Phys 2009; 36: 4059-67.

10. Palmer AJ, Brown CP, McNally EG, et al. Non-invasive imaging of cartilage in early osteoarthritis. Bone Joint J 2013; 95-B: 738-46.

11. Madry H, Kon E, Condello V, et al. Early osteoarthritis of the knee. Knee Surg Sports Traumatol Arthrosc 2016; 24: 1753-62.

12. Waldstein W, Perino G, Gilbert SL, Maher SA, Windhager R, Boettner F. OARSI osteoarthritis cartilage histopathology assessment system: a biomechanical evaluation in the human knee. J Orthop Res 2016; 34: 135-40.

13. Loeser RF. Molecular mechanisms of cartilage destruction: mechanics, inflammatory mediators, and aging collide. Arthritis Rheum 2006; 54: 1357-60.

14. Sasho T, Katsuragi J, Yamaguchi S, et al. Associations of three-dimensional T1 rho MR mapping and three-dimensional T2 mapping with macroscopic and histologic grading as a biomarker for early articular degeneration of knee cartilage. Clin Rheumatol 2017; 36: 2109-19.

15. Nebelung S, Sondern B, Oehrl S, et al. Functional MR imaging mapping of human articular cartilage response to loading. Radiology 2017; 282: 464-74.

16. Custers RJ, Creemers LB, Verbout AJ, van Rijen MH, Dhert WJ, Saris DB. Reliability, reproducibility and variability of the traditional Histologic/Histochemical Grading System vs the new OARSI Osteoarthritis Cartilage Histopathology Assessment System. Osteoarthritis Cartilage 2007; 15: 1241-8.

17. Pritzker KP, Gay S, Jimenez SA, et al. Osteoarthritis cartilage histopathology: grading and staging. Osteoarthritis Cartilage 2006; 14: 13-29.

18. Hartmann B, Marchi G, Alberton P, et al. Early detection of cartilage degeneration: a comparison of histology, fiber bragg grating-based micro-indentation, and atomic force microscopy-based nano-indentation. Int J Mol Sci 2020; 21: 7384

19. Madry H, Luyten FP, Facchini A. Biological aspects of early osteoarthritis. Knee Surg Sports Traumatol Arthrosc 2012; 20: 407-22.

20. Xu L, Servais J, Polur I, et al. Attenuation of osteoarthritis progression by reduction of discoidin domain receptor 2 in mice. Arthritis Rheum 2010; 62: 2736-44.

21. Bai H, Zhang Z, Li Y, et al. L-theanine reduced the development of knee osteoarthritis in rats via its anti-inflam- 
mation and anti-matrix degradation actions: in vivo and in vitro study. Nutrients 2020; 12: 1988.

22. Qiao YQ, Jiang PF, Gao YZ. Lutein prevents osteoarthritis through Nrf2 activation and downregulation of inflammation. Arch Med Sci 2018; 14: 617-24.

23. Luyten FP, Denti M, Filardo G, Kon E, Engebretsen L. Definition and classification of early osteoarthritis of the knee. Knee Surg Sports Traumatol Arthrosc 2012; 20: 401-6.

24. Burstein D, Gray M, Mosher T, Dardzinski B. Measures of molecular composition and structure in osteoarthritis. Radiol Clin North Am 2009; 47: 675-86.

25. Mlynárik V, Trattnig S, Huber M, Zembsch A, Imhof H. The role of relaxation times in monitoring proteoglycan depletion in articular cartilage. J Magn Reson Imaging 1999; 10: 497-502.

26. Nishioka H, Hirose J, Nakamura E, et al. T1 $\rho$ and T2 mapping reveal the in vivo extracellular matrix of articular cartilage. J Magn Reson Imaging 2012; 35: 147-55.

27. Dunn TC, Lu Y, Jin H, Ries MD, Majumdar S. T2 relaxation time of cartilage at MR imaging: comparison with severity of knee osteoarthritis. Radiology 2004; 232: 592-8.

28. Watrin A, Ruaud JP, Olivier PT, et al. T2 mapping of rat patellar cartilage. Radiology 2001; 219: 395-402.

29. David-Vaudey E, Ghosh S, Ries M, Majumdar S. T2 relaxation time measurements in osteoarthritis. Magn Reson Imaging 2004; 22: 673-82. 